\title{
MODELS OF MULTIPARAMETER BIFURCATIONS IN BOUNDARY VALUE PROBLEMS FOR ODEs OF THE FOURTH ORDER ON DIVERGENCE OF ELONGATED PLATE IN SUPERSONIC GAS FLOW
}

\author{
T.E. Badokina, Mordovian State University, Saransk, Russian Federation, \\ badokinate@gmail.com, \\ B. V. Loginov, Ulyanovsk State Technical University, Ulyanovsk, Russian Federation, \\ loginov@ulstu.ru, bvllbv@yandex.ru
}

\begin{abstract}
At the application of bifurcation theory methods to nonlinear boundary value problems for ordinary differential equations of the fourth and higher order there usually arise technical difficulties, connected with determination of bifurcation manifolds, spectral investigation of the direct and conjugate linearized problems and the proof of their Fredholm property. For overcoming of this difficulty here the roots separation method is applied to the relevant characteristic equations with subsequent presentation of critical manifolds, that allows to investigate nonlinear problems in the precise statement. Such approach is applied here to two-point boundary value problem for the nonlinear ODE of the fourth order describing the buckling (divergence) of an elongated plate in a supersonic flow of gas, subjected to compressed or extended boundary stresses at the various boundary fastenings.

Keywords: buckling of an elongated plate; bifurcation; Fredholm property.
\end{abstract}

\section{Introduction. Statement of the Problem}

Applied bifurcation problems described by ODE of the fourth order often contain various physical parameters, including several bifurcational ones. Application of Lyapunov - Schmidt method requires the precise knowledge of branching points, branching critical manifolds and zero-subspaces of the relevant linearized operators and adjoint to them. Such difficulties arise at the precise statement of problems [1, 2] on static stability loss (divergence) of a thin flexible strip-plate in supersonic flow of gas, expressed or extended by external boundary stresses and subjected by small normal load, described by boundary value problems for nonlinear ODE of the fourth order dependent on two bifurcation parameters (Mach number, compression/extension coefficient) and one small parameter. The dependence of ODE on bifurcation parameters can be expressed via roots of the relevant characteristic equation $(\mathrm{ChEq})$ of the linearized problem, which can be assumed as known precisely.

Such presentation allows to determine the critical bifurcation curves and surfaces, to construct the asymptotics of bifurcating solutions in the form of the convergent series by small deviations of bifurcation parameters on their critical values, to construct [3] the Green functions for various boundary conditions [4], first in literature, since in the well-known Melnikov's handbook [5] there was marked the absence of Green functions for aeroelasticity problems. 
In aeroelasticity problems, as a rule, the Galerkin method or grid methods are applied, often the works have only qualitative character and take into account only one bifurcation parameter - Mach number. Only in the last V.V. Bolotin works (1998 - 2005) they were considered as bifurcational. Group transformations method of T.Y. Na [6], allowing to reduce nonlinear one-parametric boundary value problem for ODE of the fourth order to the Cauchy problem was applied to the problem of strip-plate divergence in S.V.Kireev's candidate thesis [7]. The review of basic results on the divergence and flutter of plates and shells up to 1964 are given in A.S. Vol'mir monograph [1]. Contemporary review of aeroelasticity problems is contained in monograph [8].

In dimensionless variables the plate buckling is described by equation:

$$
\chi^{2}\left(\frac{w^{\prime \prime}}{\left(1+w^{2}\right)^{\frac{3}{2}}}\right)^{\prime \prime}-T w^{\prime \prime}+\beta_{0} w+\varepsilon_{3} q(x)=k K\left(w^{\prime}, M, \kappa\right)+\theta w^{\prime \prime} \int_{0}^{1}\left[\left(1+w^{2}\right)^{\frac{1}{2}}-1\right] d x
$$

General approach to solving of such type problems is considered here on the examples of boundary conditions:

(B) the left edge is free, the right one is rigidly fixed $w^{\prime \prime}(0)=w^{\prime \prime \prime}(0)=0, w(1)=$ $w^{\prime}(1)=0$;

$\left(\mathrm{B}^{\prime}\right)$ the left edge is rigidly fixed, the right one is free $w(0)=w^{\prime}(0)=0, w^{\prime \prime}(1)=$ $w^{\prime \prime \prime}(1)=0$;

(D) the left edge is fixed, the right one is rigidly fixed $w^{\prime}(0)=0, w^{\prime \prime \prime}(0)=0, w(1)=0$, $w^{\prime}(1)=0$.

Here $w=w(x)$ is the plate deflection, $0 \leq x \leq 1,-\infty<y_{1}<\infty, x=\frac{x_{1}}{d}, 0 \leq x_{1} \leq d$ are rectangular coordinates; $K\left(w^{\prime}, M, \kappa\right)=\left[1-\left(1+\frac{\kappa-1}{2} M w^{\prime}\right)^{\frac{2 \kappa}{\kappa-1}}\right]$ for one-sided flow around, $K\left(w^{\prime}, M, \kappa\right)=\left[\left(1-\frac{\kappa-1}{2} M w^{\prime}\right)^{\frac{2 \kappa}{\kappa-1}}-\left(1+\frac{\kappa-1}{2} M w^{\prime}\right)^{\frac{2 \kappa}{\kappa-1}}\right]$ for two-sided flow around; $\chi^{2}=\frac{h^{2}}{12\left(1-\mu^{2}\right) d^{2}}, T=\frac{q d}{E h}, \theta=\frac{1}{1-\mu^{2}}$ and $k=\frac{p_{0} d}{E h}$, where $d$ is the plate width, $h$ is its thickness, $E$ is the Young module, $\mu$ is the Poisson coefficient, $q<0(q>0)$ is the compressing (extending) boundary stress, $M$ is the Mach number, $p_{0}$ is the pressure and $\kappa$ is the polytropic exponent, $\beta_{0}$ is the elastic support rigidity coefficient, $\varepsilon_{3} q(x)$ is the small normal load.

For the computation of small buckled forms in neighborhoods of bifurcation parameter critical values $\left(T_{0}, M_{0}\right) ; T<0$ is the compressing, $T>0$ is the extending stress $T=T_{0}+\varepsilon_{1}$, $M=M_{0}+\varepsilon_{2}, \varepsilon_{3}=0$ methods of bifurcation and catastrophes theories [9] are applied. The presence of the small normal load is not typical for aeroelasticity problems.

This work is fulfilled in the frames of the state task N 2014 / 232 of the Russian Education and Sciences Ministry. The theme of scientific investigation work: Elaboration of mathematical methods for the investigation of dynamics and stability of deformated elements of constructions, installations, apparata and devices under aerohydrodynamical, heat and shock effects.

\section{General Model of Divergence of a Thin Elongated Elastically Supported Plate in Supersonic Flow of Gas in the Local Form}

Expansion of the nonlinearities of (1) in series on degrees of small by norm solution $w$ in a neighborhood of bifurcation parameters critical values gives it's local presentation:

$$
B w \equiv \chi^{2} w^{(4)}-T_{0} w^{\prime \prime}+\sigma_{0} w^{\prime}+\beta_{0} w=
$$




$$
\begin{gathered}
=\chi^{2}\left(\frac{3}{2} w^{\prime 2} w^{(4)}+3 w^{\prime \prime 3}+9 w^{\prime} w^{\prime \prime} w^{\prime \prime \prime}\right)-1(2) k \kappa w^{\prime} \varepsilon_{2}+\varepsilon_{1} w^{\prime \prime}-\varepsilon_{3} q(x)+\frac{\theta}{2} w^{\prime \prime} \int_{0}^{1} w^{\prime 2} d x- \\
-\left\{\begin{array}{l}
\frac{k \kappa(\kappa+1)}{4} M_{0}^{2} w^{\prime 2}+\frac{k \kappa(\kappa+1)}{2} M_{0} \varepsilon_{2} w^{\prime 2}+\frac{k \kappa(\kappa+1)}{12} M_{0}^{3} w^{\prime 3}+\ldots \\
\frac{k \kappa(\kappa+1)}{6} M_{0}^{3} w^{\prime 3}+\ldots
\end{array}\right.
\end{gathered}
$$

where the factor $1(2)$ in the parameter $\sigma$, the upper (lower) line respond to one-sided (two-sided) gas flow around the plate, and the left-hand-side of (2) together with one of the boundary conditions defines the Fredholm operator $B: C^{4+\alpha}[0,1] \rightarrow C^{\alpha}[0,1]$ with one-dimensional zero-subspace $N(B)=\operatorname{span}\{\varphi(x)\}$ and deflect-subspace $N^{*}(B)=$ $\operatorname{span}\{\psi(x)\}$.

The relevant conjugate operator $B^{*}$ is constructed by the integration by parts of the square form $\mathcal{L}(w) \cdot \omega$ along the segment $[0,1]$ taking into account the boundary conditions for the direct problem:

$$
\mathcal{L}(\omega) \equiv \chi^{2} \omega^{(4)}-T_{0} \omega^{\prime \prime}-\sigma_{0} \omega^{\prime}+\beta_{0} \omega ;
$$

$\left(\mathrm{B}^{*}\right) \chi^{2} \omega^{\prime \prime}(0)-T \omega(0)=0, \chi^{2} \omega^{(3)}(0)-T \omega^{\prime}(0)-\sigma \omega(0)=0, \omega(1)=0, \omega^{\prime}(1)=0$;

$\left(\mathrm{B}^{\prime *}\right) \omega(0)=0, \omega^{\prime}(0)=0, \chi^{2} \omega^{\prime \prime}(1)-T \omega^{\prime}(1)=0, \chi^{2} \omega^{(3)}(1)-T \omega^{\prime}(1)-\sigma \omega(1)=0$;

$\left(\mathrm{D}^{*}\right) \omega^{\prime}(0)=0, \chi^{2} \omega^{(3)}(0)-T \omega^{\prime}(0)-\sigma \omega(0)=0, \omega(1)=0, \omega^{\prime}(1)=0$.

Application of the Schmidt regularizator $\tilde{B}=B+\langle\cdot, \gamma\rangle z$, where $\gamma$ and $z$ are the biorthogonal elements to $\varphi \in N(B)$ and $\psi \in N^{*}(B)$ respectively, $\tilde{B}^{-1}=\Gamma$, with the expansion $w=w_{1000} \xi+w_{0100} \varepsilon_{1}+w_{0010} \varepsilon_{2}+w_{0001} \varepsilon_{3}+\sum_{k+|\alpha|>1} w_{k \alpha} \xi^{k} \varepsilon^{\alpha}$ give the expansion by $\xi$ and $\varepsilon$ of the E. Schmidt branching equation (BEq) $L(\xi, \varepsilon)=\xi-\langle w(\xi, \varepsilon), \gamma\rangle=0$.

For the one-sided and respectively for the two-sided flow around the plate the main part of BEqs take the forms:

$$
L(\xi, \varepsilon)=L_{2000} \xi^{2}+L_{0001} \varepsilon_{3}+L_{1001} \xi \varepsilon_{3}+L_{1100} \xi \varepsilon_{1}+L_{1010} \xi \varepsilon_{2}+\ldots=0
$$

where $L_{2000}=\frac{k \kappa(\kappa+1)}{4} M_{0}^{2}\left\langle\varphi^{\prime 2}, \psi\right\rangle, L_{0001}=-\langle q, \psi\rangle, L_{1100}=-\left\langle\varphi^{\prime \prime}, \psi\right\rangle, L_{1010}=$ $k \kappa\left\langle\varphi^{\prime}, \psi\right\rangle, L_{1001}=\frac{k \kappa(\kappa+1)}{2} M_{0}^{2}\left\langle\varphi^{\prime}(\Gamma q)^{\prime}, \psi\right\rangle$ and respectively

$$
L(\xi, \varepsilon) \equiv L_{3000} \xi^{3}+L_{0001} \varepsilon_{3}+L_{1100} \xi \varepsilon_{1}+L_{1010} \xi \varepsilon_{2}+L_{1001} \xi \varepsilon_{3}+\ldots=0
$$

where $L_{3000}=\frac{k \kappa(\kappa+1)}{6} M_{0}^{3}\left\langle\varphi^{\prime 3}, \psi\right\rangle-\chi^{2}\left\langle\frac{3}{2} \varphi^{\prime 2} \varphi^{(4)}+3 \varphi^{\prime \prime 3}+9 \varphi^{\prime} \varphi^{\prime \prime} \varphi^{\prime \prime \prime}, \psi\right\rangle-\frac{\theta}{2}\left\langle\varphi^{\prime \prime} \int_{0}^{1} \varphi^{\prime 2} d x, \psi\right\rangle$, $L_{2000}=0, L_{0001}=-\langle q, \psi\rangle, L_{1001}=0, L_{1100}=-\left\langle\varphi^{\prime \prime}, \psi\right\rangle, L_{1010}=\sigma_{0}\left\langle\varphi^{\prime}, \psi\right\rangle$.

\section{Investigation of the Roots Distribution of the ChEq for the Linearization}

To the linearized operator $B$, defined by the differential equation

$$
\mathcal{L}(w) \equiv \chi^{2} w^{(4)}-T_{0} w^{\prime \prime}+\sigma_{0} w^{\prime}+\beta_{0} w=0, \quad \sigma_{0}=1(2) k \kappa M_{0}
$$

with one of the boundary conditions responds the ChEq

$$
\lambda^{4}-a \lambda^{2}+b \lambda+c=0, \quad a=\frac{T_{0}}{\chi^{2}}, b=\frac{\sigma_{0}}{\chi^{2}}, c=\frac{\beta_{0}}{\chi^{2}} .
$$


At the investigation of ChEq the Sturm method [10] for the roots separation is used, according to which the number of the sign changes in the sequence of functions $f_{0}=$ $\lambda^{4}-a \lambda^{2}+b \lambda+c, f_{1}=f_{0}^{\prime}=4 \lambda^{3}-2 a \lambda+b, f_{2}=\frac{a}{2} \lambda^{2}-\frac{3 b}{4} \lambda-c, f_{3}=\frac{2 a^{3}-8 a c-9 b^{2}}{a^{2}} \lambda-\frac{b\left(12 c+a^{2}\right)}{a^{2}}, f_{4}=$ $\left\{\frac{f_{2}}{f_{3}}\right\}=\frac{1}{4\left(2 a^{3}-8 a c-9 b^{2}\right)}\left[4 a^{5} b^{2}-27 a^{2} b^{4}-324 b^{4} c+16 a^{6} c+256 a^{2} c^{3}+18 b^{4} c-128 a^{4} c^{2}-144 a^{3} b^{2} c\right]$ on the boundaries of some intervals says about the presence of real roots inside them. The made analysis taking into account the coefficients $b>0, c>0$ by physical meaning, shows that the ChEq has the roots of the following forms:

1. Two negative and two positive roots $\lambda_{1}=-\alpha_{1}, \lambda_{2}=-\alpha_{2}, \lambda_{3}=\alpha_{3}, \lambda_{4}=\alpha_{4} \alpha_{i}>0$ when $T>0, f_{3}^{1}=\frac{2 a^{3}-8 a c-9 b^{2}}{a^{2}}>0, f_{4}>0$;

2. Two negative and a pair of complex-conjugate roots $\lambda_{1}=-\alpha_{1}, \lambda_{2}=-\alpha_{2}, \lambda_{3,4}=$ $\gamma \pm \delta i\left(\alpha_{1}, \alpha_{2}, \gamma, \delta>0\right)$ when $2.1 T>0, f_{3}^{1}>0, f_{4}<0 ; 2.2 T>0, f_{3}^{1}<0, f_{4}<0$; $2.3 T<0, f_{3}^{1}<0, f_{4}<0$.

3. Two pairs of complex-conjugate roots $\lambda_{1,2}=-\gamma \pm \delta_{1} i, \lambda_{3,4}=\gamma \pm \delta_{2} i, \gamma, \delta_{k}>0$, if $3.1 T>0, f_{3}^{1}<0, f_{4}>0 ; 3.2 T<0, f_{3}^{1}>0, f_{4}>0 ; 3.3 T<0, f_{3}^{1}<0, f_{4}>0$.

4. Two positive and a pair of complex-conjugate roots $\lambda_{1}=\alpha_{1}, \lambda_{2}=\alpha_{2}, \lambda_{3,4}=-\gamma \pm i \delta$ $\left(\alpha_{k}, \gamma, \delta>0\right)$, if $T<0, f_{3}^{1}>0, f_{4}<0$.

Remark 1. The Sturm theorem determines the roots of the $\mathrm{ChEq}$ as independent functions of the four variables. Application of the Vieta theorem $\lambda_{1}+\lambda_{2}+\lambda_{3}+\lambda_{4}=0$ allows to reduce their number up to three in non-degenerate cases (two variables in degenerate cases).

Lemma 1. ChEq (6) hasn't got the roots of the form $4^{\circ}$.

In fact, the Vieta theorem $\alpha_{1}+\alpha_{2}-2 \gamma=0,-2 \gamma \alpha_{1} \alpha_{2}+\left(\alpha_{1}+\alpha_{2}\right)\left(\gamma^{2}+\delta^{2}\right)=b$ and the change $\alpha_{1}=2 \gamma-\alpha_{2}$ reduce the second equation to the quadratic equation with respect to $\alpha_{2}: \alpha_{2}^{2}-2 \gamma \alpha_{2}+\gamma^{2}+\delta^{2}+\frac{b}{2 \gamma}$ having negative discriminant $4 \gamma^{2}-4\left(\gamma^{2} \delta^{2}+\frac{b}{2 \gamma}\right)<0$.

In the set 1 the Vieta theorem allows to overdeterminate the roots in the following form: $\lambda_{1}=-2 \alpha-\delta_{1}, \lambda_{2}=-2 \alpha+\delta_{1}, \lambda_{3}=2 \alpha-\delta_{2}, \lambda_{4}=2 \alpha+\delta_{2}$. Corrected in such way roots of the form 2 are the following: $\lambda_{1}=-2 \gamma-\alpha, \lambda_{2}=-2 \gamma+\alpha, \lambda_{3}=\gamma-\delta$, $\lambda_{4}=\gamma+\delta$. The roots of the form 3 are dependent on three variables and does not required improvement.

Thus at the combined application of the Sturm method and the Vieta theorem the following statement can be proved.

Lemma 2. The considered ChEq can have the roots of the three following nondegenerate types: $1^{\circ} . \lambda_{1,2}=-\gamma \pm i \delta_{1}, \lambda_{3,4}=\gamma \pm i \delta_{2}\left(\gamma>0, \delta_{1} \geq \delta_{2}>0\right) ; 2^{\circ} . \lambda_{1}=-\alpha_{1}, \lambda_{2}=-\alpha_{2}$, $\lambda_{3,4}=\gamma \pm i \delta\left(\alpha_{1}>\alpha_{2}>0, \gamma, \delta>0\right) ; 3^{\circ} . \lambda_{1,2}=-\alpha \pm \beta_{1}, \lambda_{3,4}=\alpha \pm \beta_{2} \quad\left(\alpha, \beta_{1}, \beta_{2}>\right.$ $\left.0, \beta_{2}<\beta_{1}<\alpha\right)$ and three degenerate types, where there are two-multiple roots, obtained by the passage to limit from nondegenerate cases: $1^{\circ} \rightarrow 2^{\circ} . \lambda_{1,2}=-\alpha, \lambda_{3,4}=\gamma \pm i \delta$ $(\gamma>0, \delta \rightarrow 0) ; 1^{\circ} \rightarrow 3^{\circ} . \lambda_{1,2}=-\alpha, \lambda_{3,4}=\alpha(\alpha>0), \delta_{1}, \delta_{2} \rightarrow 0 ; 2^{\circ} \rightarrow 3^{\circ} . \lambda_{1}=-\alpha$ , $\lambda_{2}=-2 \gamma+\alpha, \lambda_{3,4}=\gamma(\alpha, \gamma>0, \gamma<\alpha<2 \gamma)$.

The statement of Lemma 2 can be presented on the following scheme on fig. 1 .

Lemma 3. When $\sigma \neq 0$ the characteristic equation (6) can't have the roots $1^{\circ} \rightarrow 3^{\circ}$. 


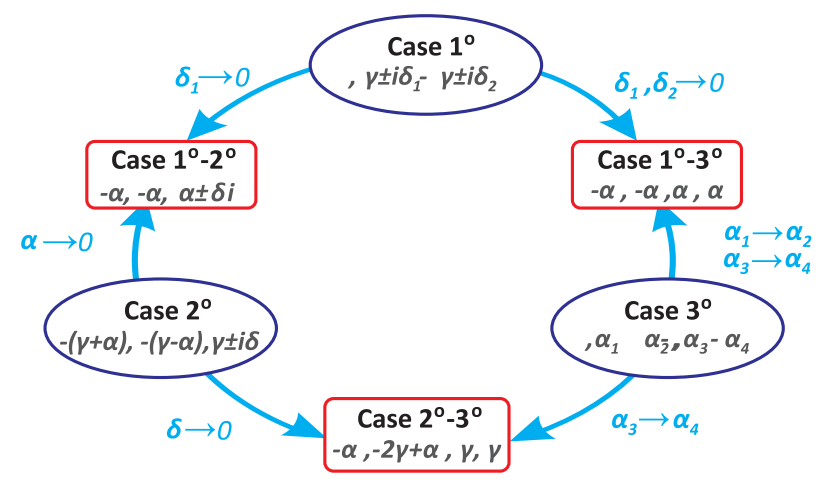

Fig. 1. Scheme of roots degeneration for the $\mathrm{ChEq}(6)$

In fact, when $\lambda_{1,2}=-\alpha$ and $\lambda_{3,4}=\alpha$, the Vieta theorem gives the following relations between $\alpha$ and the ChEq coefficients $a=2 \alpha^{2}, b=0, c=\alpha^{4}$ and consequently this equation takes the form $\left(\lambda^{2}-\alpha^{2}\right)^{2}=0$, that is possible only if $\sigma=0 \Rightarrow M=0$.

Remark 2. The presented scheme of roots degeneration allows to fulfill the checking procedure for the Green functions construction and computation of bifurcating solutions asymptotics by means of limit passage to multiple roots of ChEq.

Remark 3. The roots of ChEq for the conjugate problem (3) coincide by modulus with the roots of ChEq for the direct problem and are opposite by sign.

\section{Bifurcation Solutions Asymptotics at $\varepsilon_{3} \neq 0$}

Asymptotics of bifurcating solution on three small parameters $\varepsilon_{1}, \varepsilon_{2}, \varepsilon_{3}$ in a bifurcation point $\left(T_{0}, M_{0}, 0\right)$ is computed for the cases of the critical (bifurcation) manifolds existence, which are determined by the equality to zero of the boundary conditions matrix determinant(BCMD). For $\varepsilon_{3} \neq 0$ the main parts of BEqs (4) and (5) must be investigated.

When $L_{2000} \neq 0$ the change $\eta=\xi+\frac{L_{1100} \varepsilon_{1}+L_{1010} \varepsilon_{2}+L_{1001} \varepsilon_{3}}{L_{2000}}$ reduces BEq (4) to the form $\eta^{2}+\alpha=0$, where $\alpha=\frac{L_{0001}+L_{0101} \varepsilon_{1}+L_{0011} \varepsilon_{2}+L_{0002} \varepsilon_{3}}{L_{2000}} \varepsilon_{3}-\frac{\left(L_{1100} \varepsilon_{1}+L_{1010} \varepsilon_{2}+L_{1001} \varepsilon_{3}\right)^{2}}{4 L_{2000}}$. In the neighborhood of the branching point $\xi=0, \varepsilon_{1}=0, \varepsilon_{2}=0, \varepsilon_{3}=0$ one has $\eta= \pm \sqrt{-\alpha}$ and after the return to variables $\xi$, $\varepsilon$ the following result follows.

Theorem 1. For one-sided flow around the strip-plate when $\varepsilon_{3} \neq 0$ and $L_{2000} \neq 0$ the solution of problem (2) has the form

$$
\begin{aligned}
w(x)=\left[-\frac{L_{1100} \varepsilon_{1}+L_{1010} \varepsilon_{2}+L_{1001} \varepsilon_{3}}{L_{2000}} \pm\left(\frac{\left(L_{1100} \varepsilon_{1}+L_{1010} \varepsilon_{2}+L_{1001} \varepsilon_{3}\right)^{2}}{4 L_{2000}}-\right.\right. & \\
& \left.\left.-\frac{L_{0001} \varepsilon_{3}+\left(L_{0101} \varepsilon_{1}+L_{0011} \varepsilon_{2}\right) \varepsilon_{3}+L_{0002} \varepsilon_{3}^{2}}{L_{2000}}\right)^{\frac{1}{2}}\right] \varphi(x)+o\left(\left|\varepsilon_{1}\right|,\left|\varepsilon_{2}\right|,\left|\varepsilon_{3}\right|\right) .
\end{aligned}
$$

When $\varepsilon_{3}=0$ and $L_{2000} \neq 0$ the solution of problem (2) is presented by the series, convergent in a small neighbourhood of $\varepsilon_{1}=0, \varepsilon_{2}=0$

$$
w(x)=-\frac{\left(L_{1100} \varepsilon_{1}+L_{1010} \varepsilon_{2}\right)}{L_{2000}} \varphi(x)+o\left(\left|\varepsilon_{1}\right|,\left|\varepsilon_{2}\right|\right)
$$

i.e. the transcritical bifurcation takes place. 
Consider now the case of two-sided flow around of plate. Here the main part of the BEq has form (5).

By changing $\alpha=\frac{L_{1100} \varepsilon_{1}+L_{1010} \varepsilon_{2}}{L_{3000}}$ and $\beta=\frac{L_{0101} \varepsilon_{1}+L_{0011} \varepsilon_{2}+L_{0001}}{L_{3000}} \varepsilon_{3}$ when $L_{3000} \neq 0$ the equation (5) is rewritten in the form $L(\xi) \equiv \xi^{3}+\xi \alpha+\beta$ which hasn't got degeneration, since $L^{\prime}(\xi)=3 \xi^{2}+\alpha>0$. Therefore setting $\alpha=-\mu^{2}, \beta=v^{3}$, reduce equation (7) to the form

$$
\xi^{3}-\xi \mu^{2}+v^{3}=0
$$

the discriminant curve for which is determinated by the solution to the system

$$
\xi^{3}-\xi \mu^{2}+v^{3}=0, \quad 3 \xi^{2}-\mu^{2}=0,
$$

having the form $\mu= \pm \sqrt{3} \xi, v=2^{1 / 3} \xi$, i.e. $v= \pm B \mu, B=\frac{2^{1 / 3}}{3^{1 / 2}}<1$.

The plane of parameters $(\mu, v)$ is splitted on two domains $D_{1}$, where $\left|\frac{v}{\mu}\right|<1$ and equation (7) has three solutions, and $D_{2}$, where (7) has only one solution. In the domain $D_{1}$, supposing $v \neq 0$, divide (7) on $\mu^{3}$ and introduce new variables $\eta=\frac{\xi}{\mu}$ and $\lambda=\frac{v}{\mu}$. Then equation (7) takes the form $\eta^{3}-\eta+\lambda^{3}=0$. According to theorem on inverse function, $\eta$ is an analytic function of $\lambda^{3}$. Since for $\lambda=0$ it has the solutions $\eta=0, \eta=1, \eta=-1$, the last equation by the Newton diagram method determines the asymptotics of these three solutions: $\eta=\lambda^{3}+\lambda^{9}+3 \lambda^{15}+12 \lambda^{21}+o\left(\left|\lambda^{21}\right|\right), \eta=1-\frac{1}{2} \lambda^{3}-\frac{3}{8} \lambda^{6}+o\left(\left|\lambda^{6}\right|\right)$, $\eta=-1+\frac{1}{2} \lambda^{3}-\frac{3}{8} \lambda^{6}+o\left(\left|\lambda^{6}\right|\right)$.

The returning to the variables $\xi, \mu$ and $v$ implies the expansion of the function $\xi=\xi(\mu)$ in Taylor - Laurent - Puiseux series inside of some angular sector deleted in its top

$$
\begin{gathered}
\xi=\left(\frac{v}{\mu}\right)^{3}+\left(\frac{v}{\mu}\right)^{9}+3\left(\frac{v}{\mu}\right)^{15}+12\left(\frac{v}{\mu}\right)^{21}+\ldots \quad \text { or } \quad \xi=\frac{\beta}{\alpha}+\frac{\beta^{3}}{\alpha^{4}}+3 \frac{\beta^{5}}{\alpha^{7}}+12 \frac{\beta^{7}}{\mu^{10}}+\ldots \\
\xi=\mu-\frac{v^{3}}{2 \mu^{2}}-\frac{3 v^{6}}{8 \mu^{5}}+\ldots \quad \text { or } \quad \xi=(-\alpha)^{1 / 2}+\frac{\beta}{2 \alpha}-\frac{3 \beta^{2}}{8(-\alpha)^{5 / 2}}+\ldots \\
\xi=-\mu-\frac{v^{3}}{2 \mu^{2}}+\frac{3 v^{6}}{8 \mu^{5}}+\ldots \quad \text { or } \quad \xi=-(-\alpha)^{1 / 2}+\frac{\beta}{2 \alpha}+\frac{3 \beta^{2}}{8(-\alpha)^{5 / 2}}+\ldots
\end{gathered}
$$

Analogously for the determination of solutions asymptotics in the domain $D_{2}$, where $\left|\frac{\mu}{v}\right|<1$, divide the equation (7) on $v^{3}$ and introduce the changes $\eta=\frac{\xi}{v}$ and $\lambda=\frac{\mu}{v}$. The equation arises $\eta^{3}-\eta \lambda+1=0$, having only one solution $\eta=-1$ corresponding to $\lambda=0$. For sufficiently small $\lambda$ this solution has the asymptotics $\eta=-1-\frac{1}{3} \lambda^{2}+\frac{1}{81} \lambda^{6}+\ldots$ or $\xi=-v+\frac{\mu^{3}}{3 v^{2}}-\frac{\mu^{6}}{81 v^{4}}+\ldots$ or $\xi=-\beta^{1 / 3}+\frac{(-\alpha)^{3 / 2}}{3 \beta^{2 / 3}}-\frac{\alpha^{3}}{81 \beta^{5 / 3}}+\ldots$

On the straight lines $v= \pm B \mu$, demarcating the domains $D_{1}$ and $D_{2}$ equation (7) has the form $\xi^{3}-\xi \mu^{2} \pm B^{3} \mu^{3}=0$. The usage of the change $\eta=\frac{\xi}{\mu}$ gives two equations $\eta^{3}-\eta \pm B^{3}=0$, every of which has two solutions $\left(\frac{\sqrt{3}}{3},-\frac{2 \sqrt{3}}{3}\right)$ and $\left(-\frac{\sqrt{3}}{3}, \frac{2 \sqrt{3}}{3}\right)$, respectively $\xi= \pm \frac{\sqrt{3}}{3} \mu$ and $\xi= \pm \frac{2 \sqrt{3}}{3} \mu$ of the type $\xi \approx K(-\alpha)^{1 / 2}$.

Remark 4. At the presence of small normal load $\varepsilon_{3} \neq 0$ the functions $L(\xi, \varepsilon)$ in the BEqs both for one-sided and two-sided flow around the plate the point $\xi=0, \varepsilon=0$ is nonsingular, since $L(0,0,0,0)=0$ but $\frac{\partial L(\xi, \varepsilon)}{\partial \varepsilon_{3}} \neq 0$ implies $d L(0,0,0,0) \neq 0$, therefore a 
catastrophe is absent. In every separate case of the presented investigation of the BEqs it is not difficult to write out the solution of the nonlinear bifurcation problem. However at the absence of compression/extension load $(T=0)$ and two-sided flow around the plate the Lypounov - Shmidt BEq has the form $L\left(\xi, \varepsilon_{2}, \varepsilon_{3}\right)=L_{300} \xi^{3}+L_{101} \xi \varepsilon_{3}+L_{010} \varepsilon_{3}+\ldots=0$. Its investigation is made in [13], where it is shown that in a neighbourhood of the bifurcation point the catastrophe of the fold-type take place. At the absence of small normal load theorems 1 and 2 types are true.

The values of BEqs coefficients and respectively the asymptotics of bifurcating solutions are inconvenient and therefore are omitted here.

\section{Boundary Conditions D $\left(\varepsilon_{3}=0\right)$}

Remark 5. The divergence of the plate takes place in the cases of bifurcation (critical) manifold existence, for every case of the $\mathrm{ChEq}$ distribution we prove their absence or existence and at there existence the basic elements $\varphi \in N(B), \psi \in N^{*}(B)$ are computed. However the computation asymptotics is omitted due to it's inconvenience.

The case $\mathbf{1}^{\circ}$. ChEq (6) has two pairs of complex conjugate roots: $-\gamma \pm \delta_{1} i, \gamma \pm \delta_{2} i$. According to Vieta theorem $\delta_{1}^{2}+\delta_{2}^{2}-2 \gamma^{2}=-a, 2 \gamma\left(\delta_{1}^{2}-\delta_{2}^{2}\right)=-b, \gamma^{4}+\gamma^{2}\left(\delta_{1}^{2}+\delta_{2}^{2}\right)+\delta_{1}^{2} \delta_{2}^{2}=$ $c \Rightarrow \delta_{1}=\gamma\left(1-\frac{2}{2 \gamma^{2}}-\frac{b}{4 \gamma^{3}}\right)^{1 / 2}, \delta_{2}=\gamma\left(1+\frac{2}{2 \gamma^{2}}+\frac{b}{4 \gamma^{3}}\right)^{1 / 2}$.

To the solution of the linearized problem $w(x)=e^{-\gamma x}\left(c_{1} \cos \left(\delta_{1} x\right)+c_{2} \sin \left(\delta_{1} x\right)\right)+$ $e^{\gamma x}\left(c_{3} \cos \left(\delta_{2} x\right)+c_{4} \sin \left(\delta_{2} x\right)\right)$ the BCMD has the form

$$
\begin{gathered}
\Delta_{D}=2 \gamma \delta_{1} \delta_{2}\left(\left(\gamma^{2}+\delta_{2}^{2}\right) e^{-2 \gamma}-\left(\gamma^{2}+\delta_{1}^{2}\right) e^{2 \gamma}\right)-\left(\left(\gamma \delta_{2}^{4}+4 \gamma^{3} \delta_{2}^{2}-4 \delta_{1}^{2} \gamma^{3}-\delta_{1}^{4} \gamma\right) \sin \delta_{2}+\right. \\
\left.+\delta_{2} \cos \delta_{2}\left(\delta_{1}^{4}+\left(3 \gamma^{2}-\delta_{2}^{2}\right) \delta_{1}^{2}+4 \gamma^{4}+\gamma^{2} \delta_{2}^{2}\right)\right) \sin \delta_{1}+\delta_{1}\left(\left(\left(\gamma^{2}-\delta_{2}^{2}\right) \delta_{1}^{2}+4 \gamma^{4}+\right.\right. \\
\left.\left.+3 \gamma^{2} \delta_{2}^{2}+\delta_{2}^{4}\right) \sin \delta_{2}-2 \gamma \delta_{2} \cos \delta_{2}\left(\delta_{1}^{2}-\delta_{2}^{2}\right)\right) \cos \delta_{1}=0
\end{gathered}
$$

At the fixed values of support rigidity coefficient there exist such values of $\gamma, \delta_{1}, \delta_{2}$, for which $\Delta\left(\gamma^{1}, \delta_{1}^{1}, \delta_{2}^{1}\right) \cdot \Delta\left(\gamma^{2}, \delta_{1}^{2}, \delta_{2}^{2}\right)<0$. For example, at $c=32,237, \gamma^{1}=1, \delta_{1}^{1}=1,1971$, $\delta_{2}^{1}=3,5 ; \gamma^{2}=1, \delta_{1}^{2}=1,1972, \delta_{2}^{2}=3,5$.

Basic elements of the subspaces $N(B)$ and $N^{*}(B)$ are:

$$
\begin{gathered}
\varphi(x)=\frac{1}{\Delta_{0}}\left[\delta_{2}\left(\delta_{1} \cos \left(\delta_{1} x\right)+\gamma \sin \left(\delta_{1} x\right)\right) e^{\gamma(2-x)}+\delta_{1}\left(\delta_{2} \cos \left(\delta_{2} x\right)-\gamma \sin \left(\delta_{2} x\right)\right) e^{-\gamma(2-x)}-\right. \\
-e^{-\gamma x}\left(\left(\left(2 \gamma^{2}+\delta_{2}^{2}\right) \sin \delta_{2}-\gamma \delta_{2} \cos \delta_{2}\right) \sin \left(\delta_{1}(1-x)\right)-\delta_{1}\left(\gamma \sin \delta_{2}-\delta_{2} \cos \delta_{2}\right) \cos \left(\delta_{1}(1-x)\right)-\right. \\
\left.-e^{\gamma x}\left(\delta_{2}\left(\gamma \sin \delta_{1}+\delta_{1} \cos \delta_{1}\right) \cos \left(\delta_{2}(1-x)\right)+\left(\left(2 \gamma^{2}+\delta_{1}^{2}\right) \sin \delta_{1}+\gamma \delta_{1} \cos \delta_{1}\right) \sin \left(\delta_{2}(1-x)\right)\right)\right] \\
\psi(x)=\frac{1}{\Delta_{0}^{*}}\left[\delta_{1}\left(\gamma \sin \left(\delta_{2} x\right)+\delta_{2} \cos \left(\delta_{2} x\right)\right) e^{\gamma(2+x)}-\delta_{2}\left(\gamma \sin \left(\delta_{1} x\right)-\delta_{1} \cos \left(\delta_{1} x\right)\right) e^{-\gamma(2+x)}-\right. \\
-e^{-\gamma x}\left(\left(\left(2 \gamma^{2}+\delta_{2}^{2}\right) \sin \delta_{2}+\gamma \delta_{2} \cos \delta_{2}\right) \sin \left(\delta_{1}(1-x)\right)+\delta_{1}\left(\gamma \sin \delta_{2}+\delta_{2} \cos \delta_{2}\right) \cos \left(\delta_{1}(1-x)\right)\right)- \\
\left.-e^{\gamma x}\left(\left(\left(2 \gamma^{2}+\delta_{1}^{2}\right) \sin \delta_{1}-\gamma \delta_{1} \cos \delta_{1}\right) \sin \left(\delta_{2}(1-x)\right)-\delta_{2}\left(\gamma \sin \delta_{1}-\delta_{2} \cos \delta_{1}\right) \cos \left(\delta_{2}(1-x)\right)\right)\right],
\end{gathered}
$$

where $\Delta_{0}=\left(\left(2 \gamma^{2}+\delta_{1}^{2}\right) \cos \delta_{2}-\gamma \delta_{2} \sin \delta_{2}\right) \sin \delta_{1}+\delta_{1}\left(\gamma \cos \delta_{2}-\delta_{2} \sin \delta_{2}\right) \cos \delta_{1}-\gamma \delta_{1} e^{-2 \gamma}$, $\Delta_{0}^{*}=\left(\left(2 \gamma^{2}+\delta_{1}^{2}\right) \cos \delta_{2}+\gamma \delta_{2} \sin \delta_{2}\right) \sin \delta_{1}-\delta_{1}\left(\gamma \cos \delta_{2}+\delta_{2} \sin \delta_{2}\right) \cos \delta_{1}+\gamma \delta_{1} e^{2 \gamma}, \Delta_{0}^{*}(\gamma)=$ $\Delta_{0}(-\gamma)$ 
Remark 6. Here and further $\Delta_{0}$ and $\Delta_{0}^{*}$ are different from zero minors of the third order in the points of critical manifold $\Delta_{D}=0$ of BCMD for direct and conjugate problems.

The case $2^{\circ}$. Characteristic equation has two negative and a pair of complex-conjugate numbers: $-(\alpha+\gamma),-(\gamma-\alpha), \gamma \pm \delta i$. Here according to the Vieta theorem $\alpha_{1}+\alpha_{2}=2 \gamma$, $\alpha_{1} \alpha_{2}-2 \gamma\left(\alpha_{1}+\alpha_{2}\right)+\gamma^{2}-\delta^{2}=-a, 2 \gamma \alpha_{1} \alpha_{2}-\left(\alpha_{1}+\alpha_{2}\right)\left(\gamma^{2}+\delta^{2}\right)=-b, \alpha_{1} \alpha_{2}\left(\gamma^{2}+\delta^{2}\right)=c \Rightarrow$ $\alpha_{1,2}=\gamma\left(1 \pm \sqrt{-1+\frac{a}{2 \gamma^{2}}+\frac{b}{4 \gamma^{3}}}\right)$. Thus $\alpha_{1}=\gamma(1+u), \alpha_{2}=\gamma(1-u)$, if $0<-1+\frac{a}{2 \gamma^{2}}+\frac{b}{4 \gamma^{3}}<1$. From the condition that the sum of the roots is equal to zero the logical substitutions $\alpha_{1}=$ $\gamma+\alpha, \alpha_{2}=\gamma-\alpha$ follow. Then $a=2 \gamma^{2}+\alpha^{2}-\delta^{2}, b=2 \gamma\left(\alpha^{2}+\delta^{2}\right)$ and $c=\left(\gamma^{2}-\alpha^{2}\right)\left(\gamma^{2}+\delta^{2}\right)$.

For the deflections functions $w(x)=c_{1} e^{-\alpha_{1} x}+c_{2} e^{-\alpha_{2} x}+e^{\gamma x}\left(c_{3} \cos (\delta x)+c_{4} \sin (\delta x)\right)=$ $c_{1} e^{-(\gamma+\alpha) x}+c_{2} e^{-(\gamma-\alpha) x}+e^{\gamma x}\left(c_{3} \cos (\delta x)+c_{4} \sin (\delta x)\right)$ the BCMD in variables $\alpha, \gamma, \delta$ is equal to

$$
\begin{gathered}
\Delta_{D}=4 \gamma \alpha \delta\left(\left(\gamma^{2}+\delta^{2}\right) e^{-2 \gamma}-\left(\gamma^{2}-\alpha^{2}\right) e^{2 \gamma}\right)-(\gamma-\alpha)\left(\left(4 \gamma^{3} \alpha-4 \gamma^{2} \delta^{2}-\gamma \alpha\left(\alpha^{2}+\delta^{2}\right)-\right.\right. \\
\left.\left.-\delta^{2}\left(\alpha^{2}+\delta^{2}\right)\right) \sin \delta-\delta\left(4 \gamma^{3}+4 \gamma^{2} \alpha+\gamma\left(\delta^{2}+\alpha^{2}\right)-\alpha\left(\alpha^{2}+\delta^{2}\right)\right) \cos \delta\right) e^{-\alpha}- \\
-(\gamma+\alpha)\left(\left(4 \gamma^{3} \alpha+4 \gamma^{2} \delta^{2}-\gamma \alpha\left(\alpha^{2}+\delta^{2}\right)+\delta^{2}\left(\alpha^{2}+\delta^{2}\right)\right) \sin \delta+\right. \\
\left.+\delta\left(4 \gamma^{3}-4 \gamma^{2} \alpha+\gamma\left(\delta^{2}+\alpha^{2}\right)+\alpha\left(\alpha^{2}+\delta^{2}\right)\right) \cos \delta\right) e^{\alpha}=0
\end{gathered}
$$

and determines the critical bifurcation curves. Numerical experiment shows the bifurcation points existence, where $\Delta_{D}=0$. At the fixed values of support rigidity coefficient there exist such values $(\gamma, \alpha, \delta)$, for which $\Delta\left(\alpha^{1}, \gamma^{1}, \delta^{1}\right) \cdot \Delta\left(\alpha^{2}, \gamma^{2}, \delta^{2}\right)<0$. For example at $c_{0}=$ $26,502, \alpha^{1}=2, \delta_{1}^{1}=1,5, \delta_{2}^{1}=3,33827 ; \alpha^{2}=2, \delta_{1}^{2}=1,51, \delta_{2}^{2}=3,37773$.

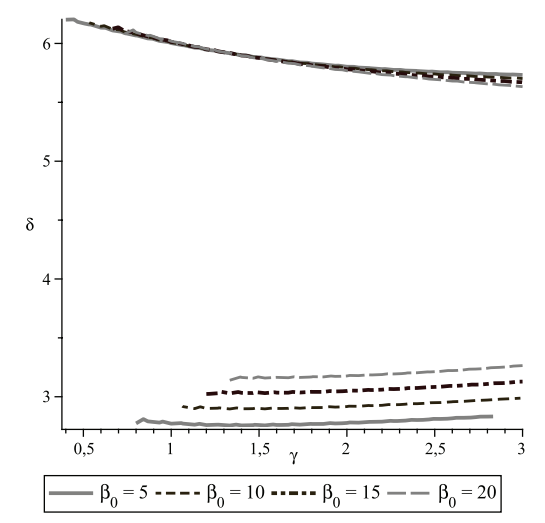

a)

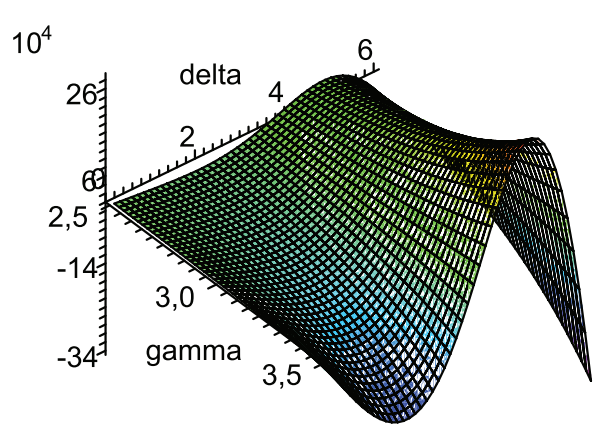

b)

Fig. 2. a) Visualisation $\Delta_{D}$ in case $1^{\circ}$; b) Relief $\Delta_{D}$ in case $2^{\circ}$ for $c=20$

Indicate here the basic elements $\varphi$ and $\psi$ of the subspaces $N(B)$ and $N^{*}(B)$

$$
\begin{gathered}
\varphi(x)=\frac{1}{\Delta_{0}}\left[\left(\left(\left(2 \gamma^{2}+\gamma \alpha+\delta^{2}\right) \sin \delta-\delta(\gamma+\alpha) \cos \delta\right) e^{-\alpha}+\delta(\gamma+\alpha) e^{2 \gamma}\right) e^{-(\gamma-\alpha) x}-\right. \\
-\left(\left(\left(2 \gamma^{2}-\gamma \alpha+\delta^{2}\right) \sin \delta-\delta(\gamma-\alpha) \cos \delta\right) e^{\alpha}+\delta(\gamma-\alpha) e^{2 \gamma}\right) e^{-(\gamma-\alpha) x}+ \\
+\left((\gamma-\alpha)((2 \gamma+\alpha) \sin (\delta(1-x))+\delta \cos (\delta(1-x))) e^{-\alpha}+2 \alpha(\gamma \sin (\delta x)-\delta \cos (\delta x)) e^{-2 \gamma}-\right. \\
\left.\left.-(\gamma+\alpha)((2 \gamma-\alpha) \sin (\delta(1-x))+\delta \cos (\delta(1-x))) e^{\alpha}\right) e^{\gamma x}\right],
\end{gathered}
$$




$$
\begin{gathered}
\psi(x)=\frac{-1}{\Delta_{0}^{*}}\left[( \gamma - \alpha ) \left(\left(\gamma\left(\alpha^{2}+2 \gamma \alpha-\delta^{2}\right) \sin (\delta)-\delta\left(2 \gamma \alpha+\alpha^{2}+\delta^{2}+2 \gamma^{2}\right) \cos (\delta)\right) e^{-\gamma}+\right.\right. \\
\left.+2 \gamma \delta(\gamma+\alpha) e^{\gamma+\alpha}\right) e^{-(\gamma-\alpha) x}+(\gamma+\alpha)\left(2 \delta \gamma(\gamma-\alpha) e^{\gamma-\alpha}+\left(\left(\alpha^{2}-2 \gamma \alpha+\delta^{2}+2 \gamma^{2}\right) \delta \cos (\delta)-\right.\right. \\
\left.\left.-\left(\alpha^{2}-2 \gamma \alpha-\delta^{2}\right) \gamma \sin (\delta)\right) e^{-\gamma}\right) e^{-(\gamma+\alpha) x}-\left(( \gamma - \alpha ) \left(\gamma\left(\alpha^{2}+2 \gamma \alpha-\delta^{2}\right) \sin (\delta x)-\delta\left(2 \gamma^{2}+\right.\right.\right. \\
\left.\left.+2 \gamma \alpha+\alpha^{2}+\delta^{2}\right) \cos (\delta x)\right) e^{\gamma-\alpha}-4 \gamma \alpha\left(\gamma^{2}+\delta^{2}\right) \sin (\delta(1-x)) e^{-\gamma}+\left(\delta \left(2 \gamma^{2}-2 \gamma \alpha+\delta^{2}+\right.\right. \\
\left.\left.\left.\left.+\alpha^{2}\right) \cos (\delta x)-\gamma\left(\alpha^{2}-2 \gamma \alpha-\delta^{2}\right) \sin (\delta x)\right) e^{\gamma+\alpha}(\gamma+\alpha)\right) e^{\gamma x}\right]
\end{gathered}
$$

where $\Delta_{0}=(\gamma-\alpha)(\delta \sin \delta-(2 \gamma+\alpha) \cos \delta) e^{-\alpha}-2 \gamma \alpha(\gamma+\alpha)((2 \gamma-\alpha) \cos \delta-\delta \sin \delta) e^{\alpha}$, $\Delta_{0}^{*}=\gamma\left((\gamma+\alpha)\left(\alpha^{2}-2 \gamma \alpha-\delta^{2}\right) e^{\gamma+\alpha}-(\gamma-\alpha)\left(\alpha^{2}+2 \gamma \alpha-\delta^{2}\right) e^{\gamma-\alpha}-4 \alpha\left(\gamma^{2}+\delta^{2}\right) e^{-\gamma} \cos \delta\right)$.

The case $3^{\circ}$ of two negative and two positive roots of ChEq (6). From the Vieta theorem it follows that $\beta_{1}>\beta_{2}, \beta_{1}<\alpha$. Consequently $a=\beta_{1}^{2}+\beta_{2}^{2}+2 \alpha^{2}, b=2 \alpha\left(\beta_{1}^{2}-\beta_{2}^{2}\right)$ and $c=\left(\alpha^{2}-\beta_{1}^{2}\right)\left(\alpha^{2}-\beta_{2}^{2}\right)$. It means, that the indicated case is possible only at the presence of compressing boundary stress. At the fixed value $c=c_{0}$ one has the relation $\alpha=\sqrt{\frac{\beta_{1}^{2}+\beta_{2}^{2}+\sqrt{\left(\beta_{1}^{2}-\beta_{2}^{2}\right)+4 c_{0}}}{2}}$. To the solution $w(x)=c_{1} e^{-\left(\alpha+\beta_{1}\right) x}+c_{2} e^{-\left(\alpha-\beta_{1}\right) x}+c_{3} e^{\left(\alpha-\beta_{2}\right) x}+$ $c_{4} e^{\left(\alpha+\beta_{2}\right) x}$ there responds a determinant $\Delta_{B}$ of the boundary condition matrix

$$
\begin{gathered}
\Delta_{D}=8 \alpha \beta_{1} \beta_{2}\left(\left(\alpha^{2}-\beta_{2}^{2}\right) e^{-2 \alpha}-\left(\alpha^{2}-\beta_{1}^{2}\right) e^{2 \alpha}\right)+\left(\alpha-\beta_{1}\right) e^{-\beta_{1}}\left(( \alpha + \beta _ { 2 } ) ( \beta _ { 1 } + \beta _ { 2 } ) \left(4 \alpha^{2}-\right.\right. \\
\left.\left.-\left(\beta_{1}-\beta_{2}\right)^{2}\right) e^{-\beta_{2}}-\left(\alpha-\beta_{2}\right)\left(\beta_{1}-\beta-2\right)\left(4 \alpha^{2}-\left(\beta_{1}+\beta_{2}\right)^{2}\right) e^{\beta_{2}}\right)+\left(\alpha+\beta_{1}\right) e^{\beta_{1}}((\alpha+ \\
\left.\left.+\beta_{2}\right)\left(\beta_{1}-\beta-2\right)\left(4 \alpha^{2}-\left(\beta_{1}+\beta_{2}\right)^{2}\right) e^{-\beta_{2}}-\left(\alpha-\beta_{2}\right)\left(\beta_{1}+\beta-2\right)\left(4 \alpha^{2}-\left(\beta_{1}-\beta_{2}\right)^{2}\right) e^{\beta_{2}}\right)=0 .
\end{gathered}
$$

Lemma 4. On the considered set $\Omega=\left\{\left(\alpha, \beta_{1}, \beta_{2}\right) \mid \beta_{1} \in(0, \alpha), \beta_{2} \in(0, \alpha), \beta_{1}>\beta_{2}\right\}$ the divergence is absent.

Proof. Introduce $\Delta_{1}=8 \alpha \beta_{1} \beta_{2}\left(\alpha^{2}-\beta_{2}^{2}\right) e^{-2 \alpha}, \Delta_{2}=\left(\alpha-\beta_{1}\right) e^{-\beta_{1}}\left(\left(\alpha+\beta_{2}\right)\left(\beta_{1}+\beta-2\right)\left(4 \alpha^{2}-\right.\right.$ $\left.\left.\left(\beta_{1}-\beta_{2}\right)^{2}\right) e^{-\beta_{2}}, \Delta_{3}=-\left(\alpha-\beta_{1}\right)\left(\alpha-\beta_{2}\right)\left(\beta_{1}-\beta-2\right)\left(4 \alpha^{2}-\left(\beta_{1}+\beta_{2}\right)^{2}\right) e^{-\beta_{1}+\beta_{2}}\right), \Delta_{4}=$ $\left(\alpha+\beta_{1}\right) e^{\beta_{1}}\left(\alpha+\beta_{2}\right)\left(\beta_{1}-\beta-2\right)\left(4 \alpha^{2}-\left(\beta_{1}+\beta_{2}\right)^{2}\right) e^{-\beta_{2}}, \Delta_{5}=-\left(\alpha+\beta_{1}\right) e^{\beta_{1}}\left(\alpha-\beta_{2}\right)\left(\beta_{1}+\beta-\right.$ 2) $\left(4 \alpha^{2}-\left(\beta_{1}-\beta_{2}\right)^{2}\right) e^{\beta_{2}}$ and $\Delta_{6}=-8 \alpha \beta_{1} \beta_{2}\left(\alpha^{2}-\beta_{1}^{2}\right) e^{2 \alpha}$ for the parts of determinant $\Delta_{D}$, containing as cofactor exponents in various degrees. Then $\Delta=\sum_{i=1}^{6} \Delta_{i}$. The restrictions implying from the Vieta theorem $e^{\beta_{1}-\beta_{2}}<e^{\beta_{1}},-e^{-\beta_{1}+\beta_{2}}<-e^{-\beta_{1}}, e^{-\beta_{1}-\beta_{2}}<e^{-\beta_{1}}$ and $-e^{\beta_{1}+\beta_{2}}<-e^{\beta_{1}}$ give the inequality $\Delta_{2}+\Delta_{3}+\Delta_{4}+\Delta_{5}<2\left(\alpha+\beta_{1}\right) e^{\beta_{1}}\left(-4 \alpha^{3} \beta_{2}+4 \alpha^{2} \beta_{1} \beta_{2}-\right.$ $\left.\alpha \beta_{1}^{2} \beta_{2}-\beta_{1}^{3} \beta_{2}+\beta_{1} \beta_{2}^{3}+\alpha \beta_{2}^{3}\right)+2\left(\alpha-\beta_{1}\right) e^{-\beta_{1}}\left(4 \alpha^{3} \beta_{2}+4 \alpha^{2} \beta_{1} \beta_{2}+\alpha \beta_{1}^{2} \beta_{2}-\beta_{1}^{3} \beta_{2}+\beta_{1} \beta_{2}^{3}-\alpha \beta_{2}^{3}\right)$. The inequalities $-e^{\beta_{1}}<-1, e^{-\beta_{1}}<1$ and $e^{\beta_{1}}$ together with negativity of coefficient before $e^{\beta_{1}}$ imply $\Delta_{2}+\Delta_{3}+\Delta_{4}+\Delta_{5}<8 \alpha \beta_{1} \beta_{2}\left(\beta_{2}^{2}-\beta_{1}^{2}\right)<0$.

For the remaining part of $\Delta_{D}$ from the inequalities $\Delta_{1}+\Delta_{6}<8 \alpha \beta_{1} \beta_{2}\left(\left(\alpha^{2}-\beta_{2}^{2}\right) e^{-2 \alpha}-\right.$ $\left.\left(\alpha^{2}-\beta_{1}^{2}\right) e^{2 \alpha}\right)$ by virtue of the inequalities $e^{-2 \alpha}<1$ and $-e^{2 \alpha}<-1$ it follows $\Delta_{1}+\Delta_{6}<$ $8 \alpha \beta_{1} \beta_{2}\left(\beta_{1}^{2}-\beta_{2}^{2}\right)<0$. The summing gives finally $\Delta<8 \alpha \beta_{1} \beta_{2}\left(\beta_{1}^{2}-\beta_{2}^{2}\right)+8 \alpha \beta_{1} \beta_{2}\left(\beta_{2}^{2}-\beta_{1}^{2}\right)=0$, i.e. $\Delta<0$ and the divergence is absent.

Further the degenerate cases are considered.

The relevant degenerate case $\mathbf{1}^{\circ}-\mathbf{2}^{\circ}$, when ChEq (6) has a two-multiple negative root and a pair of complex-conjugate roots: $-\alpha,-\alpha, \alpha \pm \delta i$. According to the Vieta theorem 
the coefficients of the ChEq are the following $a=2 \alpha^{2}-\delta^{2}, b=2 \alpha \delta^{2}, c=\alpha^{2}\left(\alpha^{2}+\delta^{2}\right)$ and the relation $\alpha>\frac{\delta}{\sqrt{2}}$ is true. For the deflections function $w(x)=c_{1} e^{-\alpha x}+c_{2} x e^{-\alpha x}+$ $e^{\alpha x}\left(c_{3} \cos (\delta x)+c_{4} \sin (\delta x)\right)$ the boundary conditions matrix determinant has the form

$$
\begin{gathered}
\Delta_{D}=2 \alpha \delta\left(\left(\alpha^{2}+\delta^{2}\right) e^{-2 \alpha}-\alpha^{2} e^{2 \alpha}\right)-\left(4 \alpha^{4}+4 \alpha^{3} \delta^{2}+3 \alpha^{2} \delta^{2}+\alpha \delta^{4}+\delta^{4}\right) \sin \delta- \\
-\alpha \delta\left(4 \alpha^{3}+\alpha \delta^{2}+2 \delta^{2}\right) \cos \delta
\end{gathered}
$$

The critical bifurcation curve consists of the points $(\alpha, \delta)$, where $\Delta=0$. At the fixed values of support rigidity coefficient $c$ there exist such values $\alpha, \delta$, on which $\Delta\left(\alpha^{1}, \delta^{1}\right) \cdot \Delta\left(\alpha^{2}, \delta^{2}\right)<$ 0 ; for example when $c=17,554, \alpha^{1}=1,25, \delta^{1}=3,11, \alpha^{2}=1,253, \delta^{2}=3,1$ and the divergence takes place.

Basic elements of $N(B)$ and $N^{*}(B)$ are the following

$$
\begin{aligned}
& \varphi(x)= \frac{1}{\Delta_{0}}\left[\left(\delta(1+\alpha x) e^{2 \alpha}-\left(2 \alpha^{2}(1-x)+\delta(1-x)-\alpha\right) \sin \delta+\delta(\alpha(1-x)-1) \cos \delta\right) e^{-\alpha x}+\right. \\
&\left.+\left(e^{-2 \alpha}(\delta \cos (\delta x)-\alpha \sin (\delta x))-\alpha(1+2 \alpha) \sin (\delta(1-x))-\delta(1+\alpha) \cos (\delta(1-x))\right) e^{\alpha x}\right] \\
& \psi(x)= \frac{1}{\Delta_{0}^{*}}\left[\left(2\left(\alpha^{2}+\delta^{2}\right)(-\alpha \sin (\delta(1-x))+\delta \cos (\delta(1-x))) e^{-\alpha}+e^{\alpha}\left(\delta \left(2 \delta^{2}+\alpha \delta^{2}+2 \alpha^{3}+\right.\right.\right.\right. \\
&\left.\left.\left.+2 \alpha^{2}\right) \cos (\delta x)\right)+\alpha\left(2 \alpha^{2}+\delta^{2}+\alpha \delta^{2}\right) \sin (\delta x)\right) \alpha e^{-\alpha x}-\left(\left[\left(3 \alpha^{3} \delta^{2} x+\delta^{4}+2 \alpha^{4}+\right.\right.\right. \\
&\left.\left.\left.\left.+\alpha^{2} \delta^{2}+\alpha \delta^{4} x\right) \sin \delta+\alpha^{3} \delta(\alpha x-1) \cos \delta\right] e^{-\alpha}+2 \alpha^{3} \delta(1+\alpha(1-x)) e^{\alpha}\right) e^{\alpha x}\right],
\end{aligned}
$$

where $\Delta_{0}=-\alpha e^{-2 \alpha}+\alpha(2 \alpha+1) \cos \delta-\delta(\alpha+1) \sin \delta$ and $\Delta_{0}^{*}=2 \alpha\left(\alpha^{2}+\delta^{2}\right)(\alpha \cos \delta+$ $\delta \sin \delta) e^{-\alpha}-\alpha^{2} e^{\alpha}\left(2 \alpha^{2}+2 \delta^{2}+\alpha \delta^{2}\right)$.

The case $\mathbf{2}^{\circ}-\mathbf{3}^{\circ}$, when ChEq (6) has the roots: $-\alpha,-(2 \gamma-\alpha)$ and $\gamma$ of the multiplicity 2. The Vieta theorem shows, that here $a=\alpha^{2}-2 \gamma \alpha+3 \gamma^{2}, b=2 \gamma(\gamma-\alpha)^{2}$, $c=\alpha \gamma^{2}(2 \gamma-\alpha)$. This is possible only for the extension boundary stresses $a>0$.

To the solution $w(x)=c_{1} e^{-\alpha x}+c_{2} e^{-(2 \gamma-\alpha) x}+c_{3} e^{\gamma x}+c_{4} x e^{\gamma x}$ the BCMD responds

$$
\Delta_{D}=4 \gamma(\alpha-\gamma)\left(\gamma^{2} e^{-2 \gamma}-\alpha(2 \gamma-\alpha) e^{2 \gamma}\right)+\alpha e^{\alpha-\gamma}\left(3 \gamma^{4}-(\alpha+8) \gamma^{3}+3 \alpha(1-\alpha) \gamma^{2}+\right.
$$$$
\left.+\alpha^{2}(\alpha+2) \gamma-\alpha^{3}\right)-(2 \gamma-\alpha) e^{-\alpha+\gamma}\left(3 \gamma^{4}+(2-\alpha) \gamma^{3}-\alpha(3 \alpha+1) \gamma^{2}+\alpha^{2}(\alpha+4) \gamma-\alpha^{3}\right)=0 .
$$

Lemma 5. On the considered set $\Omega=\{(\alpha, \gamma) \mid \alpha \in(\gamma, 2 \gamma)\}$ the divergence is absent.

Proof. In fact, according to the Vieta theorem $\alpha=\gamma+\sqrt{\gamma^{2}-\frac{c}{\gamma^{2}}}, \gamma^{4}>c$. The usage of the change $\alpha=\gamma+\tau$, where $\tau=\sqrt{\gamma^{2}-\frac{c}{\gamma^{2}}}<\gamma$ reduces the BCMD $\Delta_{D}$ to the form

$$
\begin{gathered}
\Delta_{D}=4 \gamma \tau\left(\gamma^{2} e^{-2 \gamma}-(\gamma+\tau)(\gamma-\tau) e^{2 \gamma}\right)+(\gamma+\tau) e^{\tau}\left(3 \gamma^{4}-(\gamma+\tau+8) \gamma^{3}+3(\gamma+\tau)(1-\right. \\
\left.-\gamma-\tau) \gamma^{2}+(\gamma+\tau)^{2}(\gamma+\tau+2) \gamma-(\gamma+\tau)^{3}\right)-(\gamma-\tau) e^{-\tau}\left(3 \gamma^{4}+(2-\gamma-\tau) \gamma^{3}-\right. \\
\left.-(\gamma+\tau)(3 \gamma+3 \tau+1) \gamma^{2}+(\gamma+\tau)^{2}(\gamma+\tau+4) \gamma-(\gamma+\tau)^{3}\right)= \\
=4 \tau \gamma^{3} e^{-2 \gamma}-4 \gamma \tau\left(\gamma^{2}-\tau^{2}\right) e^{2 \gamma}-(\gamma+\tau)\left(4 \gamma^{3}-4 \gamma^{3} \tau+\gamma \tau^{2}+\tau^{3}-4 \gamma^{2} \tau-\gamma \tau^{3}\right) e^{\tau}+ \\
+(\gamma-\tau)\left(4 \gamma^{3}-4 \gamma^{3} \tau+\gamma \tau^{2}-\tau^{3}+4 \gamma^{2} \tau+\gamma \tau^{3}\right) e^{-\tau}
\end{gathered}
$$

The simple inequalities $4 \tau \gamma^{3} e^{-2 \gamma}<4 \tau \gamma^{3},-4 \gamma \tau\left(\gamma^{2}-\tau^{2}\right) e^{2 \gamma}<-4 \gamma \tau\left(\gamma^{2}-\tau^{2}\right), e^{\tau}>1$ and $e^{-\tau}<1$ imply the estimate $\Delta_{D}: \Delta_{D}<4 \tau \gamma^{3}-4 \gamma \tau\left(\gamma^{2}-\tau^{2}\right)-(\gamma+\tau)\left(4 \gamma^{3}-4 \gamma^{3} \tau+\right.$ 
$\left.\gamma \tau^{2}+\tau^{3}-4 \gamma^{2} \tau-\gamma \tau^{3}\right)+(\gamma-\tau)\left(4 \gamma^{3}-4 \gamma^{3} \tau+\gamma \tau^{2}-\tau^{3}+4 \gamma^{2} \tau+\gamma \tau^{3}\right)=-2 \tau \gamma^{2}\left(4 \gamma^{2}-\tau^{2}\right)<0$. Hence $\Delta_{D}<0$ everywhere on $\Omega$ and the divergence is absent.

Remark 7. For all degenerate cases the verification of all results concerning the Green functions and asymptotics of bifurcating solutions their verification is made with the aid of limit passages from non-degenerate cases.

\title{
References
}

1. Vol'mir A.S. Ustoychivost' deformiruemykh sistem [Stability of Deformated Systems]. Moscow, Nauka, 1967. 984 p.

2. Bolotin V.V. Nekonservativnye zadachi teorii uprugoy ustoychivosti [Nonconservative Problems of the Elastic Stability Theory]. Moscow, GIFML, 1961. 339 p.

3. Naimark M.A. Lineynye differentsial'nye operatory [Linear differential operators]. Moscow, Nauka, 1969. 528 p.

4. Loginov B., Badokina T., Makeeva O. Green Functions Construction for Divergence Problems in Aeroelasticity. ROMAI Journal, 2008, vol. 4, no. 2, pp. 33-44.

5. Mel'nikov Yu.A. Influence Functions and Matrices. Ser. Text and Reference Books. Mech. Engng. Vol. 119. N.Y., Basel, Marcel Dekker, 1999. 469 p.

6. Na T.Y. Computational Methods in Engineering Boundary Value Problems. London, Academic Press, 1979. 294 p.

7. Vel'misov P.A., Kireev S.V. Matematicheskoe modelirovanie $v$ zadachakh staticheskoy neustoychivosti uprugikh elementov konstruktsiy pri aerogidrodinamicheskom vozdeystvii [Mathematical Modelling in Problems of Static Instability of Elastic Structural Elements under the Aerohydrodynamic Impact]. Ulyanovsk, UlGTU, 2011. 200 p.

8. Algazin S.D., Kiyko I.A. Flatter plastin $i$ obolochek [Flutter of plates and shells]. Moscow, Nauka, 2006. 247 p.

9. Vainberg M.M., Trenogin V.A. Teoriya vetvleniya resheniy nelineynykh uravneniy [Branching Theory of Solutions to Nonlinear Equations]. Moscow, Nauka, 1969. 524 p.

10. Shafarevich I.R. O reshenii uravneniy vysshikh stepeney (metod Shturma) [On the Resolving of Higher Degrees Equations (Sturm Method)]. Moscow, GITTL, 1987. 24 p.

Received March 11, 2015

УДК 517.927.4

DOI: $10.14529 / \mathrm{mmp} 150202$

\section{МОДЕЛИ МНОГОПАРАМЕТРИЧЕСКИХ БИФУРКАЦИЙ В КРАЕВЫХ ЗАДАЧАХ ДЛЯ ОДУ ЧЕТВЕРТОГО ПОРЯДКА О ДИВЕРГЕНЦИИ УДЛИНЕННОЙ ПЛАСТИНЫ В СВЕРХЗВУКОВОМ ПОТОКЕ ГАЗА}

\author{
Т.Е. Бадокина, Б.В. Логинов
}

При применении методов теории бифуркации в нелинейных краевых задач для обыкновенных дифференциальных уравнений четвертого и более высоких порядков, 
как правило, возникают технические трудности, связанные с определением бифуркационных многообразий, спектральным исследованием прямых и сопряженньг линеаризованных задач и доказательством их фредгольмовости. Для их преодоления применяется метод разделения корней соответствующих характеристических уравнений с последующим представлением через них критических многообразий, что позволяет исследовать нелинейные проблемы в точной постановке. Такой подход применяется здесь к двухточечной краевой задаче для нелинейных ОДу четвертого порядка, описывающих выпучивание (дивергенцию) удлиненной пластины в сверхзвуковом потоке газа при пограничном сжатии/растяжении при различных граничных закреплениях.

Ключевые слова: выпучивание удлиненной пластины; бифуркация; фредгольмовость.

\section{Литература}

1. Вольмир, А.С. Устойчивость деформируемых систем / А.С. Вольмир. - М.: Наука, 1967. $-984 \mathrm{c}$.

2. Болотин, В.В. Неконсервативные задачи теории упругой устойчивости / В.В. Болотин. М.: ГИФМЛ, 1961. - 339 с.

3. Наймарк, М.А. Линейные дифференциальные операторы / М.А. Наймарк. - М.: Наука, 1969. - $528 \mathrm{c}$.

4. Loginov, B. Green Functions Construction for Divergence Problems in Aeroelasticity / B. Loginov, T. Badokina, O. Makeeva // ROMAI Journal. - 2008. - V. 4, № 2 - P. 33-44.

5. Mel'nikov, Yu.A. Influence Functions and Matrices / Yu.A. Mel'nikov. - N.Y., Basel, Marcel Dekker, 1999. - 469 p. - (Ser. Text and Reference Books. Mech.Engng. V. 119).

6. Na, T.Y. Computational Methods in Engineering Boundary Value Problems / T.Y. Na.London: Academic Press, 1979. - 294 p.

7. Вельмисов П.А. Математическое моделирование в задачах статической неустойчивости упругих элементов конструкций при аэрогидродинамическом воздействии / П.А. Вельмисов, С.В. Киреев. - Ульяновск: УлГТУ, 2011. - 200 с.

8. Алгазин, С.Д. Флаттер пластин и оболочек / С.Д. Алгазин, И.А. Кийко. - М.: Наука, 2006. -247 c.

9. Вайнберг, М.М. Теория ветвления решений нелинейных уравнений / М.М. Вайнберг, В.А. Треногин. - М.: Наука, 1969. - 524 с.

10. Шафаревич, И.Р. О решении уравнений высших степеней (метод Штурма) / И.Р. Шафаревич. - М.: ГИТТЛ, 1954. - 24 с.

Татьяна Евгеньевна Бадокина, ассистент, кафедра «Фундаментальная информатика», Мордовский государственный университет (г. Саранск, Российская Федерация), badokinate@gmail.com.

Борис Владимирович Логинов, доктор физико-математических наук, профессор, кафедра «Высшая математика», Ульяновский государственный технический университет (г. Ульяновск, Российская Федерация), loginov@ulstu.ru, bvllbv@yandex.ru.

Поступила в редакцию 11 марта 2015 г. 